Análisis de la utilización de redes sociales por parte de instituciones culturales durante la pandemia: casos Museo de Arte de Lima y Gran Teatro Nacional Analysis of the Use of Social Networks by Cultural Institutions During the Pandemic: Cases Museo de Arte de Lima and Gran Teatro Nacional

Especialista en comunicaciones dentro del sector cultural, con más de cinco años de experiencia en creación de estrategias digitales enfocadas en la promoción de las artes visuales. Es magíster en Dirección de Proyectos Culturales por la Université Sorbonne Nouvelle de París y magíster en Historia del Arte y Curaduría por la Pontificia Universidad Católica del Perú. Actualmente, cursa un MBA en Marketing Digital \& Business - Art et Culture en l'École des Métiers de la Communication (EFAP) de París. 



\title{
Análisis de la utilización de redes sociales por parte de instituciones culturales durante la pandemia: casos Museo de Arte de Lima y Gran Teatro Nacional Analysis of the Use of Social Networks by Cultural Institutions During the Pandemic: Cases Museo de Arte de Lima and Gran Teatro Nacional
}

\author{
Susana Navarro Hospinal \\ Université Sorbonne Nouvelle, Francia \\ susana.navarro.hospinal@sorbonne-nouvelle.fr, nhsusana@gmail.com \\ (https://orcid.org/o000-0002-5968-7957)
}

Recibido: 31-05-2021 / Aceptado: 16-09-2021

https://doi.org/10.18800/conexion.202102.001

\section{PALABRAS CLAVE / KEYWORDS}

Pandemia, COVID-19, comunicación digital, institución cultural, Museo de Arte de Lima, Gran Teatro Nacional / pandemic, COVID-19, digital communication, cultural institution, Museo de Arte de Lima, Gran Teatro Nacional

\section{RESUMEN}

Las herramientas digitales no son algo nuevo, pero la pandemia ha hecho visible la necesidad de las instituciones culturales de establecer nuevas alternativas para mantenerse cerca de su público; en este escenario, lo digital no solo se ha convertido en una herramienta, sino que ha pasado a ser vital para la presencia de las artes en general. Según el Conseil International des Musées (ICOM), la comunicación no suele ser una prioridad para las organizaciones culturales, y menos aún cuando se trata de lo digital, concretamente de las redes sociales (2019, p. 5). Se realizó un análisis de las respuestas digitales de dos instituciones peruanas, el Museo de Arte de Lima (MALI) y el Gran Teatro Nacional (GTN), para establecer cómo evolucionó su presencia en redes sociales y en la web, todo en el contexto de la pandemia y de los confinamientos. Para ello, también se conversó con las personas encargadas de las áreas de comunicación de dichas instituciones.

\section{ABSTRACT}

Digital tools are not new, but the pandemic has made visible the need for cultural institutions to establish new alternatives and digital has not only become a tool, but has become vital for the empowerment of museums and culture in general. According to Conseil International des Musées (ICOM), communication is 
often not a priority for cultural organizations, and even less so when it comes to digital, specifically social networks (2019, p. 5). An analysis was made of the digital responses of two Peruvian institutions, the Museo de Arte de Lima (MALI) and the Gran Teatro Nacional (GTN), to determine how their presence in social networks and on the web evolved. For this purpose, we also talked to the people in charge of the communication areas of these institutions.

\section{Análisis de la utilización de redes sociales por parte de instituciones culturales durante la pandemia: casos Museo de Arte de Lima y Gran Teatro Nacional}

La pandemia ha afectado todos los sectores, y el cultural es uno de los que ha visto canceladas o transformadas sus actividades. Ello ha hecho incluso más notoria la necesidad de establecer nuevas alternativas creativas en la implementación de las herramientas digitales, lo que es una parte vital para fortalecer la presencia de las instituciones culturales.

No es nuevo que distintas instituciones han ido explorando cada vez más las herramientas digitales y sus grandes posibilidades para tratar de unir la experiencia digital y la física. Según el ICOM, la comunicación no suele ser una prioridad para las organizaciones culturales, y menos aún cuando se trata de lo digital, concretamente de las redes sociales
(Conseil International des Musées, 2019, p. 5).

El presente artículo analiza las respuestas digitales de dos instituciones peruanas, el Museo de Arte de Lima (MALI) y el Gran Teatro Nacional (GTN), frente a la pandemia y el confinamiento, esto para conocer cómo evolucionó su presencia digital en redes sociales, si es que hubo una transformación en su relación con el público y cómo se reajustaron los equipos de comunicación de manera interna. Con este objetivo, se conversó con las personas encargadas de las áreas de comunicación de ambas instituciones.

Es importante tener en cuenta el concepto de marca, que está vinculado con el prestigio; en un contexto normal, aporta calidad y confianza, así como estatus, no solo en los sectores económicos tradicionales, sino también en las instituciones culturales. Un adecuado posicionamiento de marca ha permitido a algunas de estas instituciones afrontar mejor la pandemia.

«La creatividad es el principal motor de la economía del arte», menciona De Garay (2008). En el sector cultural, estamos ante un sistema económico en el que lo dominante es la oferta, es decir, la creación artística, la cual se encuentra en el centro junto con el artista; establecer un vínculo con el público en el medio cultural es vital y este factor es lo primero que se ha modificado por las circunstancias de la pandemia. 


\section{Las instituciones culturales en el Perú y el público}

El informe 22 indicadores de la cultura para el desarrollo en Perú. Resumen analítico de Perú, realizado por el Ministerio de Cultura con el apoyo de la Organización de las Naciones Unidas para la Educación, la Ciencia y la Cultura (2015), señala que las actividades culturales en el Perú representan el $1.58 \%$ del PBI nacional y emplean al $3.3 \%$ de la población (p. 12).

El informe Hacia un museo sostenible. Oferta y demanda de los museos y centros expositivos de Lima, hecho por el Museo de Arte de Lima en el año 2018, encontró que una de las mayores fortalezas de los museos son sus colecciones, gracias a las cuales han logrado un conocimiento especializado; se han esforzado por llevar a cabo diversas iniciativas que pueden ayudar en áreas como la profesionalización del sector, la educación, la inclusión social y la retención de la audiencia.

Entre las conclusiones del estudio del MALI, se descubrió que el conocimiento y el estudio del público se hace a través del registro de visitantes; sin embargo, son pocas las instituciones que realmente hacen un análisis del perfil de la concurrencia:

La principal consecuencia es que el sector se encuentra divorciado de su público en gran medida, por lo que los programas, proyectos y eventos que desarrollan se realizan sin contar con las necesidades, opiniones e intereses del público y de su comunidad circundante (Museo de Arte de Lima, 2018, p. 141).

Hay pocos estudios que examinen el consumo cultural en el Perú. Los pocos que existen no toman en cuenta todas las expresiones artísticas, las diferentes instituciones culturales, o no consideran todo el territorio nacional. Pero es importante entender cómo es que el público peruano llega a tomar la decisión de asistir a un evento cultural y, en nuestro caso, de ir a un museo o teatro.

El Ministerio de Cultura del Perú publicó un estudio sobre cómo fue el consumo de ciertas líneas culturales, específicamente en el caso del teatro, entre los años 2016 y 2019 a nivel nacional. Se encontró, entre otra información, lo siguiente:

- En cuanto al teatro, el aumento de la asistencia entre 2016 y 2019 fue solo del $0.9 \%$. Sin embargo, se observa que el incremento más significativo se produjo en las zonas rurales (Ministerio de Cultura, 2021, p. 13).

- Al menos el $50 \%$ de la población que asistió a un teatro en 2019 lo hizo porque la entrada era gratuita, a diferencia del $37.6 \%$ que compró una entrada (pp. 8 y 13). 
- El $32.4 \%$ de las personas que asistieron a un espectáculo en el 2019 tienen al menos un título de posgrado (p. 11).

- El grupo de encuestados que afirma no haber asistido al teatro por falta de interés varía entre el 40 $\%$ y el $50 \%$ (p. 12).

Otro estudio publicado en abril de 2020 y preparado por el Instituto de Opinión Pública de la Pontificia Universidad Católica del Perú sobre la frecuencia de asistencia a eventos culturales públicos en las áreas metropolitanas de Lima y Callao, que incluye cifras desde el año 2009 hasta el 2019, mostró que casi el $70 \%$ de la población declaró no tener mucho tiempo libre que pueda dedicar al consumo de productos culturales. Por otro lado, en cuanto a la asistencia, más del $70 \%$ de los encuestados no visitó una exposición de arte, pintura, fotografía o escultura en el año 2019, mientras que la cifra fue del $79 \%$ para las obras de teatro y el $66 \%$ en el caso de los espectáculos de danza. En el mismo estudio, el MALI se encuentra en la quinta posición entre la asistencia a diversos museos, y muestra una disminución de $9 \%$ entre el año 2009 y el 2019 (Instituto de Opinión Pública, 2020).

Al leer estos estudios, es más fácil entender que el consumo cultural en el Perú no solo está influido por la facilidad de cas, sino que las actividades culturales no son vistas como unas que puedan retribuir de algún modo al espectador, de manera que valga la pena el esfuerzo de acercarse a ellas. También se observa esto en el estudio realizado por el Instituto Nacional de Estadística e Informática en 2019, en el que se señala que las razones para no visitar un museo están entre la falta de interés $-33.8 \%-$ y la falta de tiempo $-42.5 \%-$, mientras que, en el teatro, los porcentajes llegan a $48.3 \%$ para el primer motivo y a $26.5 \%$ para el segundo.

En la fase cualitativa del informe del Museo de Arte de Lima (2018), se constató que, para el público nacional, aunque los museos y centros culturales tienen como objetivo preservar y difundir la cultura, esta sigue percibiéndose como elitista: se la asocia a artes tradicionales como la ópera, el ballet, la música clásica y las artes plásticas, y se cree que pertenece solo a un grupo social concreto. Por otro lado, se menciona que los no visitantes también participan en actividades culturales, pero que buscan formatos menos serios, como también lo muestra el estudio realizado por el Instituto Nacional de Estadística e Informática (2019), en el que las fiestas tradicionales - patronales, carnavales, etcétera-y las funciones de cine son las que ocupan los primeros puestos de asistencia. Entre las razones para visitar el museo, encontramos, en primer lugar, la de enriquecer conocimientos culturales $-60 \%-$; 
en segundo lugar, la búsqueda de una nueva experiencia $-48 \%-$; y, en tercer lugar, la curiosidad $-36 \%-$ (Museo de Arte de Lima, 2018, p. 160).

Entre las conclusiones, se ve que ya antes de la pandemia se conocía que se debía empoderar más al visitante a través de una mayor interacción con actividades en las que el espectador no sea solo un receptor de información: «El acceso a la información está mediado por la interacción, por lo que buscan [la audiencia] una persona que los oriente, pero a su propio ritmo» (Museo de Arte de Lima, 2018, p. 213).

En junio de 2020, el Ministerio de Cultura y la Municipalidad de Lima presentaron un informe sobre el impacto de la pandemia en las instituciones culturales. Este documento resalta que, entre marzo y junio de ese año, las pérdidas económicas alcanzaron los S/ 162967928 soles, y que el $91 \%$ de las personas entrevistadas eran trabajadores autónomos y mypes.

Sin embargo, a pesar de las dificultades, según el mismo informe, el $53 \%$ de los encuestados cree que es posible virtualizar sus actividades, entre los cuales el $40 \%$ emplearía Facebook; el $19 \%$, YouTube; y el $18 \%$, Instagram (Ministerio de Cultura y Municipalidad de Lima, 2020). No obstante, también es necesario notar que todavía existe un porcentaje importante de instituciones culturales que no logran digitalizar sus contenidos.

\section{Lo digital en las instituciones culturales del Perú y la pandemia}

Cuando hablamos de lo digital, solemos pensar en las redes sociales, pero también se trata de la digitalización de contenidos y fuentes de información, que va desde la creación y el mantenimiento de la página web hasta la producción de contenidos en diversos formatos. La cantidad de recursos es muy variada, y no todos tienen la misma funcionalidad y objetivos.

Para tener el contexto sobre la brecha digital en el Perú, en la encuesta de la Asociación Peruana de Empresas de Inteligencia de Mercados sobre el nivel socioeconómico, publicada en octubre de 2020, encontramos lo siguiente:

- $\quad$ En Lima Metropolitana, el 58.7 $\%$ de los hogares tiene conexión a internet. Dentro de este grupo, el $100 \%$ del nivel socioeconómico A cuenta con este servicio; en el nivel B, el $95 \%$; en el C, el $67.2 \%$; en el D, el 17.2\%; y, en el E, el $5.3 \%$ (Asociación Peruana de Empresas de Inteligencia de Mercados, 2020, p. 47).

- A nivel nacional, la cifra se reduce: el $45 \%$ de los hogares cuenta con conexión a internet. El 100 \% del nivel socioeconómico A tiene el servicio; en el $B$, el $94.3 \%$; en el C, el 68.2\%; en el D, el 18.3\%; y, en el E, el 2.9 \% (Asociación Pe- 
ruana de Empresas de Inteligencia de Mercados, 2020, p. 58).

Hootsuite (2021) calculó que, en enero de 2021, iba a haber 19.9 millones de usuarios de internet en el Perú, lo que supone un aumento del $13 \%$ de los usuarios con respecto a 2020. Sin embargo, las cifras más resaltantes son que hubo 27 millones de usuarios de redes sociales para enero de 2021, lo que representa un aumento del $13 \%$ en comparación con el año anterior y da un total de $81.4 \%$ en relación con la población total del país. Otro dato para tener en cuenta es que, según la misma fuente, en el Perú había 36.14 millones de conexiones móviles en enero de 2021, lo cual representa el $109 \%$ de la población; esto se debe a que hay personas que cuentan con más de una conexión móvil.

Si bien existe una deficiencia en lograr un mayor número de conexiones a internet en los hogares, las conexiones móviles son las que han remplazado dicha deficiencia y las redes sociales son ampliamente utilizadas por la población; son elementales como fuente de información y un medio de comunicación con el que se espera dialogar, discutir y una interacción centrada en el usuario (Choque Porras, 2018, p. 94).

Ahora, pasando a las instituciones museales, en el caso de Lima, Choque Porras (2018) explica que Facebook es la red social más utilizada, aunque solo el $77 \%$ una y tres publicaciones diarias. Twitter es la segunda red social más utilizada $-37 \%-$; YouTube, la tercera $-34 \%-$; e Instagram, la cuarta $-11 \%-$. En su investigación, la autora concluye que las instituciones son conscientes de sus problemas o deficiencias en cuanto a la presencia en las redes sociales, pero que muchos de los museos analizados no entienden realmente todo el potencial de las redes sociales; piensan que no son espacios de interacción o que se trata solo de plataformas en las que se vierten contenidos sin más análisis ni intención de lograr interacción. En muchos casos, la interacción con el usuario no se produce porque se piensa que no es necesaria o porque no se sabe qué responder.

Lo digital ya ha cambiado nuestros hábitos y ha modificado la forma de consumir arte $\mathrm{o}$, al principio, la manera de acercarse a él. Los museos y las instituciones culturales no deben limitarse a utilizar las redes sociales o las plataformas digitales en general, sino que deben integrarlas en el ritual de visita al museo:

Las nuevas tecnologías están modificando las maneras de comportarse de las personas y su entorno cambia continuamente. En los tiempos actuales no se trata de ofertar productos culturales, lo que hay que entender muy bien, es lo que significa y qué factores influyen en la búsqueda para obtenerlo (Leal Jiménez y Quero Gervilla, 2011, p. 120). 
En el Museo de Arte de Lima (2018), ya se había concluido que, aunque la mayoría de los museos tienen presencia en la web y en las redes sociales, el sector aún está lejos de estar digitalizado. Las herramientas digitales son todavía ajenas a muchas de estas instituciones, y destaca la falta de interés por conseguir una apertura digital de las colecciones.

El mismo informe menciona que, en cuanto a comunicación digital, en el caso de los 50 museos encuestados, 35 utilizan su página web, 38 tienen Facebook y solo 15 usan otras redes sociales como Twitter, Instagram, blogs, etcétera. En cuanto a Facebook, que es el medio de promoción digital más importante para los museos, 12 museos no tienen página de Facebook; 5 publican más de una vez al día; 12, una vez al día; 9, dos o tres veces por semana; 3, semanalmente; 2 , dos veces al mes; y 3, mensualmente. En cuanto a los visitantes y a saber cómo se informan de los eventos o exposiciones, $47 \%$ respondió que fue a través de las páginas web y el $47 \%$ lo hizo por intermedio de las redes sociales (Museo de Arte de Lima, 2018, pp. 97, 100 y 175).

Todo esto nos demuestra que se tiene a una ciudadanía que está acostumbrada a las redes sociales y a informarse por medios digitales, y que todo esto es consultado por medio de los teléfonos móviles. Pero también se evidencia que hay instituciones culturales que han dejado de lado su presencia digital, y la pande- mia hizo que esta deficiencia fuera más grande y el desafío por contrarrestar, más fuerte.

\section{La respuesta digital del MALI y del GTN}

Al conversar con los encargados de las secciones de comunicaciones de las dos instituciones, se pudo encontrar que se desarrollaron tres etapas. Cada una marca una evolución en las repuestas digitales.

\section{Primera etapa}

La primera etapa estuvo marcada por un tiempo de aprendizaje de cómo se trabajaría en confinamiento y por las primeras reacciones dentro del MALI y del GTN en cuanto a la gestión de la comunicación externa e interna. Las dos instituciones debían ahora comunicarse solo vía virtual y, además, había que conocer qué contenidos estaban o no digitalizados.

Facebook es, sin duda, la red social más utilizada por el MALI y el GTN, y la que mejores resultados obtuvo en la primera etapa. En el caso del MALI, está claro que los objetivos de la campaña fueron, en primer lugar, que el público viera que el museo se convertía en una institución que acompañaba durante este difícil periodo y mostrar que se contaba con recursos digitales que podían ser utilizados desde casa. En el caso del GTN, se deseaba mantener al público informado de la reprogramación de actividades, la cance- 
lación de eventos y la devolución de las entradas compradas. Para el teatro, este periodo solo duró la primera semana del confinamiento; al mismo tiempo, también se estaban preparando los contenidos que se presentarían en la segunda etapa de la estrategia, como veremos en la siguiente sección.

En el MALI, se utilizaron publicaciones en las que se mostraban piezas de su colección con mensajes divertidos y lúdicos, pero estas solo duraron poco tiempo y no se volvieron a repetir. También se realizaron publicaciones en las que pedían al público que recreara obras de arte con objetos de casa, esto como la campaña realizada por el Getty Museum -\#GettyMuseumChallenge-, pero esta iniciativa tampoco se continuó, a pesar de que este tipo de publicaciones generan un mejor engagement (Figura 1).

Es importante ver que los hashtags son diferentes. En el museo hay un uso de diferentes hashtags. El primero que se utilizó fue \#QuédateEnCasa (Figura 2); luego, \#MALIEnCasa. Esto último ocurrió por la necesidad de crear un hashtag que contenga el nombre del museo. Después, se utilizó un tercer hashtag: \#QuédateEnCasaChallenge. Se utilizaron tres hashtags al mismo tiempo y, aunque se entiende que los tres pertenecen a la misma campaña, esto genera que el público no se sienta identificado con ninguno de ellos. El poder que podría generar el hashtag como simple forma de reconocimiento

Figura 1

Publicación del MALI en Facebook durante la primera etapa de la pandemia

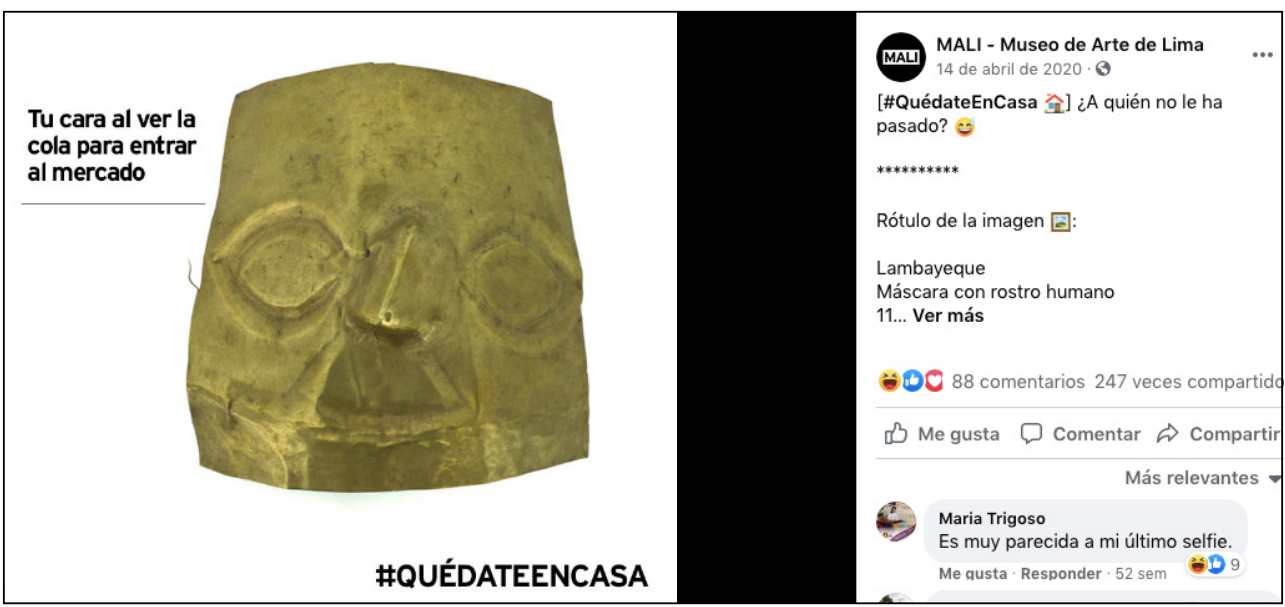

Nota. Captura de pantalla de [\#QuédateEnCasa] ¿A quién no le ha pasado? [Imagen adjunta] [Actualización de estado], por MALI - Museo de Arte de Lima, 14 de abril de 2020, Facebook (https://shorturl.at/amxX4). 
Figura 2

Publicación del MALI en Facebook con uso de hashtags

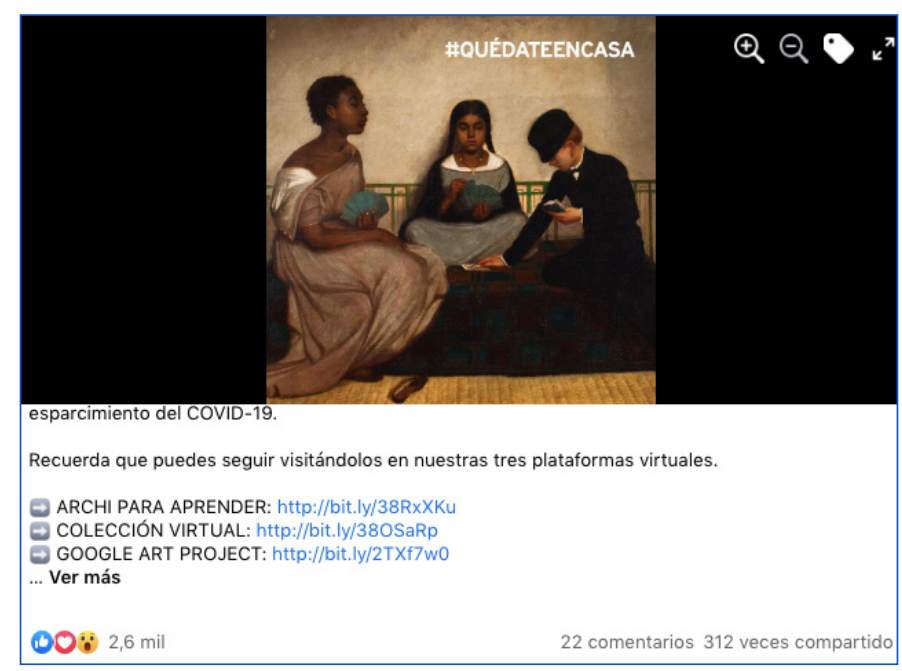

Nota. Captura de pantalla de \#QuédateEnCasa. Recuerda que puedes seguir visitándolos en nuestras tres plataformas virtuales [Imagen adjunta] [Actualización de estado], por MALI - Museo de Arte de Lima, marzo de 2021, Facebook (https://shorturl.at/ijwV8).

se diluye y los contenidos no se sienten unificados bajo una misma etiqueta. Por eso, si se quiere hacer campañas que pidan la participación del público, con esa multiplicidad de hashtags, no se tendrá la misma respuesta.

Por otro lado, el teatro utilizó \#YoMeQuedoEnCasa, que es un hashtag utilizado por el Gobierno peruano en todas sus plataformas, por lo que no es uno que el Gran Teatro Nacional haya utilizado de manera particular. La mayoría de las publicaciones y el uso de este hashtag fueron piezas creadas por el Ministerio de Salud, el Gobierno central o el Ministerio de Cultura, que se alineaban con un mismo mensaje a nivel estatal.
Más adelante, empezaron a promocionar los programas emitidos en la cadena de televisión pública -TV Perú-, que se incluyó en el logotipo del teatro y en el hashtag. Aunque este tipo de publicaciones no cesaron del todo, solo más tarde, con la generación de nuevos contenidos propios del teatro, las redes del GTN se hicieron más variadas y centradas en la vocación cultural de la institución.

\section{Segunda etapa}

Esta etapa es la que abarca más tiempo, ya que en ese momento se empezaron a generar contenidos y cada institución comenzó a tener más actividades propias. En el caso del MALI, se inició la campaña 
\#SubastaMALI, para la cual se realizaron tres tipos de publicaciones: en Facebook, se presentaron los afiches de los conversatorios; en Instagram, se divulgaron videos en forma de invitación que mostraban a los artistas, los cuales se publicaban el mismo día del conversatorio; luego, se transmitía el video en vivo en Facebook, que finalmente se colgaba en YouTube. Este fue un ciclo grande, ya que la subasta estaba prevista para octubre.

En el caso del GTN, no existió realmente una campaña específica sobre un programa especial o una temporada concreta, sino que se centró en la promoción de la plataforma GTN en Vivo, la cual se creó específicamente para agrupar los contenidos digitales en video del teatro (Figura 3).

Por otro lado, Patricia Villanueva, jefa de Estrategias de Comunicación del MALI, mencionó que, para la creación de los videos, pidieron a los participantes que enviaran sus audios; luego, el equipo del museo editaba los videos agregando imágenes. Se trataba de una forma diferente de generar contenidos, considerando que la gente no podía reunirse físicamente y que asistir al museo no era posible.

En ningún momento se utilizó la opción de streaming de video de Instagram - IGTVpara hacer una conversación o presentar

Figura 3

Publicación en Facebook del Gran Teatro Nacional

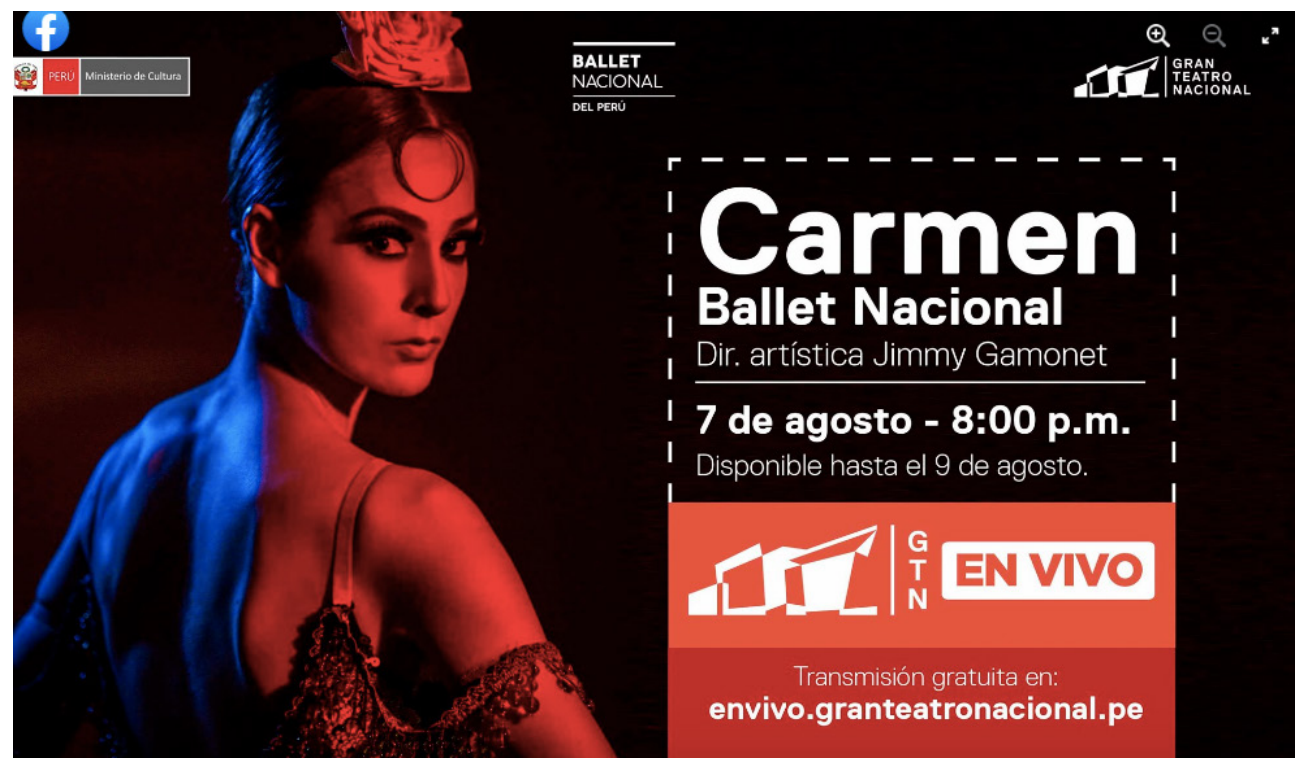

Nota. Captura de pantalla de Carmen. Ballet Nacional. Dir. artística Jimmy Gamonet [Imagen], por Gran Teatro Nacional, 30 de abril de 2021, Facebook (https://shorturl.at/blyY0). 
un video más largo. El contenido de esa plataforma es breve y directo. Como se mencionó anteriormente, en esta campaña se puede apreciar una mejor diferenciación del contenido de acuerdo con la audiencia de cada plataforma y podemos ver que se trató de hacer una publicación intercalada y diferenciar el contenido, esto para no saturar al público con una misma publicación en todas las plataformas, sobre todo cuando el programa de conversatorios era muy extenso.

En el caso del Gran Teatro Nacional, se empezó por la emisión de programas pregrabados. Ello trajo consigo varios problemas: primero, no contaban con todos los espectáculos grabados; $\mathrm{y}$, segundo, su publicación en plataformas digitales también implicaba todo un nuevo aspecto en derechos de autor, algo que el GTN no había tenido la necesidad de tomar en cuenta antes. Algunas de las grabaciones podían ser difundidas en las redes sociales y en la televisión nacional; otras solo tenían permiso para ser publicadas durante un tiempo definido en el sitio web de GTN en Vivo. Esto ocasionaba que fuese imposible que un gran número de programas estuviesen disponibles indefinidamente, y no podían publicarse en todas las plataformas, lo que habría maximizado la cantidad de audiencia.

Luego, se pasó a la creación de contenidos propios, esto debido a que se aligeraban las restricciones y ya era posible grabar videos dentro del teatro. Así, se propusieron programas como Café concierto o El teatro por dentro. Para ello, las alianzas fueron importantes: los programas producidos por el GTN también podían ser vistos por redes sociales -Facebook - y por Cultura24.tv - plataforma del Ministerio de Cultura-, y, además, se contaba con la difusión en TV Perú. Si bien estos últimos medios son del Estado, solo a partir de la pandemia se comprobó la necesidad y la importancia de unificar esfuerzos para poder llegar a un público más extenso.

Es importante señalar que el uso de esta plataforma, GTN en Vivo, y de las redes sociales, con especial atención a Facebook, ha conseguido descentralizar la demanda de productos culturales. El teatro como espacio físico se encuentra en Lima; sin embargo, podemos ver que, en el estudio realizado en mayo de 2020 , en el que se muestran cifras preliminares sobre el público que ve GTN en Vivo, hay un gran público de provincias como Cuzco, Arequipa, Piura o Lambayeque, e incluso del extranjero, como España, Argentina y Colombia (Gran Teatro Nacional, 2020).

Por otro lado, aunque Facebook es la red social más utilizada por el GTN, este también ha decidido aventurarse en nuevas redes. En este punto, el teatro no parece utilizar todavía todos los recursos que ofrecen las demás redes sociales, como el caso de Twitter, que se convierte en un medio puramente informativo, pero en el 
que no hay actividad ni interacción con el público. Comparando con la segunda red social más utilizada, Instagram, que sí tiene un mayor flujo de información, en esta sí se genera contenido especial para las historias - stories - y para los carretes -reels-, y se ve un interés por hacer crecer esta red social.

En un segundo paso, constatamos un mejor uso de los hashtags por parte del MALI al intentar agrupar los contenidos mostrando un consenso sobre cuáles se utilizarán y cómo. Esto ayuda a encontrar las publicaciones reunidas bajo un mismo hashtag, aunque sean compartidas por otros usuarios o páginas, y, además, también permite una mejor interconectividad entre plataformas.

En este punto es importante destacar que, en el caso del MALI, hubo una falta de respuesta a las preguntas o comentarios realizados en sus redes, independientemente de la plataforma. Por ejemplo, diversos talleres y clases del museo fueron cancelados y no pudieron ser reprogramados. A raíz de ello, empezó a aumentar el número de comentarios que preguntaban cómo se haría el reembolso, ya que muchos usuarios no querían tomar las clases de forma virtual. Es posible que las respuestas a los comentarios se hayan hecho internamente; sin embargo, es importante que, al menos con un simple mensaje público, el usuario sepa que su comentario o pregunta ha sido tomado en cuenta.
En comparación, el GTN siempre responde un mensaje o comentario; es parte de la atención al cliente. Por otro lado, por ser una organización que forma parte del Estado, se entiende que busque satisfacer los gustos de un público muy diverso y trate de dar espacio a todas las artes. No obstante, esto puede resultar abrumador si no se tiene una programación clara. Por eso me parece relevante que hayan optado, en las diversas plataformas, por el recordatorio constante de estrenos y transmisiones.

\section{Tercera etapa}

La tercera etapa estuvo marcada por la inclusión de nuevas herramientas digitales. Es importante entender que la aplicación de estas es un proceso de aprendizaje sobre cuáles funcionan mejor y cuáles no.

En el caso del MALI, esta etapa empezó con la decisión del museo de abrir de nuevo las puertas a los visitantes para que se puedan ver las exposiciones «Khipus. Nuestra historia en Nudos», disponible del 5 de noviembre de 2020 al 25 de abril de 2021; y «De un punto a otra», de Flavia Gandolfo, expuesta del 12 de noviembre de 2020 al 16 de mayo de 2021. La mayoría de publicaciones fueron realizadas en la plataforma de Facebook y no todos los contenidos fueron compartidos en Instagram o en Twitter, lo cual muestra la poca integración entre las diferentes redes sociales.

Esta etapa es interesante, ya que no se dejó de construir contenido para las redes 
sociales; se prepararon pódcast, se incluyeron entrevistas, se hizo contenido para Spotify y -algo más importante- se optó por digitalizar las salas de exposición: ahora era posible visitar la exposición en formato $3 \mathrm{D}$.

Como en la etapa anterior, se puede ver un mejor uso de los hashtags. Se siguió haciendo campañas pequeñas con motivos específicos, como la \#SemanaDelAmor, \#MALIAdentro, entre otros. Estos hashtags permitieron agrupar mejor los contenidos e hicieron que sean de fácil reconocimiento. Por otro lado, los diseños de cada una de las publicaciones mejoraron e, incluso, se diferenció entre las plataformas.

En el caso del GTN, como nueva plataforma se incorporó TikTok, en el cual se utilizaron los mismos reels de Instagram, y estos tuvieron un buen desempeño, es decir, respuestas positivas por parte de los usuarios. Por otro lado, el teatro decidió usar un newsletter, el cual los ayuda a segmentar a su público de acuerdo con su preferencia por la danza, el teatro o la música.

En esta etapa, en el caso del MALI, fue la primera vez que se vio una participación directa de los mediadores, lo que generó una relación más estrecha con el público, siempre bajo una misma temática según la campaña; además, ellos mismos pidieron la participación del público mediante concursos.

\section{Conclusiones}

El MALI y el GTN son dos instituciones que, antes de la pandemia, contaban con los insumos necesarios para poder generar contenidos. Si bien, en un principio, tuvieron que organizarse y cambiar su forma de trabajar, debido al confinamiento y a la imposibilidad de reunirse, lograron proponer contenido pertinente que les permitió mantenerse cerca de su público.

Según estudios previos a la pandemia, ya se conocía que las instituciones culturales tenían un problema en la implementación de estrategias digitales que las ayuden a comunicarse de manera efectiva con su público. Se conocían las deficiencias, ocasionadas por diversos motivos, como falta de personal especializado, falta de presupuesto para la digitalización de colecciones, entre otros. La pandemia ahondó en la brecha entre las instituciones que pudieron afrontar mejor el cierre temporal de sus puertas y aquellas que no lograron mantener una presencia en redes.

El GTN y el MALI se enfrentaron, en un principio, a una oleada de mensajes en los que se pedía claridad sobre la devolución de entradas, reembolsos, etcétera. En este momento, el GTN tuvo una respuesta rápida y clara, mientras que el MALI se demoró en mostrar una política de respuesta frente a las dudas de la audiencia. Esto es una muestra de que es importante tener 
una respuesta de comunicación efectiva y rápida frente a una crisis, y de que, ante la incertidumbre, no debe ser una opción el no responder nada; es necesario recordar que las redes sociales influyen significativamente en la imagen de la institución.

En cuanto a la audiencia del GTN, en la gestión de las redes sociales en general no se encuentra una clara segmentación de su audiencia. La clasificación de los públicos es quizá más visible con el boletín de noticias - newsletter-, en el que se pide que se mencione un género artístico preferido. Y, si bien el teatro buscaba dar una oferta variada, el público del GTN suele preferir los espectáculos que tienen un ángulo folclórico o tradicional, ya sea en la música o en la danza; esto es algo que funciona muy bien como una forma de introducir nuevos públicos a una oferta más variada y a la que no están acostumbrados.

$\mathrm{Al}$ inicio de la pandemia, el GTN ya contaba con una fuerte presencia en medios digitales. Recién en el inicio de la pandemia y el confinamiento, se hicieron alianzas estratégicas con otras plataformas del propio Estado. Es necesario que las instituciones culturales analicen y construyan alianzas con diversas instituciones o empresas, asociaciones que les permitan desarrollar proyectos no solo digitales, sino también presenciales y de manera permanente.

El MALI empezó con contenido que fun- acciones positivas y comentarios, pero se quedó en solo dos o tres publicaciones, cuando podría haber generado más compromiso con el público. El desafío del museo a lo largo de las tres etapas ha sido más el de saber cómo interactuar con el usuario y lograr incentivar este intercambio. El GTN se halló en el lado opuesto: se mostró muy activo en redes, no solo en los comentarios y respuestas, sino generando reacciones o introduciendo datos curiosos, también en las trasmisiones en vivo. El problema es que solo una o dos personas en el equipo se encargaban de esto, lo que generaba una carga laboral muy fuerte para un equipo que tenía poca gente y que era responsable de muchas actividades.

Es importante destacar, en el caso del GTN, que todas las emisiones, independientemente de la plataforma utilizada, se transmitieron de forma gratuita. Esta es una manera de cumplir el objetivo de democratizar el arte, pero es lógico pensar que el teatro también debe tener un contenido que genere beneficios. Cuando se le preguntó al responsable del espacio de comunicación por qué no pensaban en cobrar por los contenidos que transmitían, mencionó que era porque las grabaciones que tenían estaban pensadas para archivo y contenían «errores». Por eso, de cara al futuro, se espera que esto pueda cambiar y que se piense en espectáculos no solo para ver físicamente en el teatro, sino que también se abran a las posibilidades de entrar en el negocio en línea. 
Por otro lado, el MALI y el GTN son dos instituciones que ya gozan de un gran reconocimiento en el Perú, con una marca de confianza construida a lo largo del tiempo. El estatus de ambas organizaciones las ha hecho más fuertes para afrontar los retos de la pandemia en términos de herramientas e insumos para crear sus contenidos digitales, como es el caso de la digitalización de la colección del MALI y el registro en video de las presentaciones pasadas en el GTN.

Los responsables de comunicación con los que hablamos para esta investigación ${ }^{1}$ mencionaron que, en sus respectivas instituciones, se había producido un cambio de mentalidad: las diversas plataformas digitales se habían convertido en una necesidad y en herramientas que ofrecían diversas oportunidades, desde la información hasta la mediación entre las artes y el público. Aunque ambos expresaron su interés en que esto continúe en el futuro, se espera que las distintas iniciativas puedan mantenerse en términos de creatividad y presupuesto.

Si bien no hay un estudio específico que explore la situación de diversas instituciones culturales en las redes sociales, es igual de necesario conocer cómo la brecha digital que existe entre estas instituciones ha afectado su respuesta ante la pandemia.

${ }^{1}$ Patricia Villanueva, jefa de Estrategias de Comunicación del Museo de Arte de Lima; y Vasco Nuñez Cuba, jefe del área de Comunicaciones del Gran Teatro Nacional. 


\section{REFERENCIAS}

Asociación Peruana de Empresas de Inteligencia de Mercados. (2020, octubre). Niveles socioeconómicos 2020. http://apeim.com.pe/wp-content/ uploads/2020/10/APEIM-NSE-2020. pdf

Choque Porras, A. (2018). Situación de las redes sociales en los museos del Perú: claves y desafíos en Lima, Arequipa y Cusco. En B. Calderón Roca, A. Choque Porras y F. Quiles García (Eds.), Nuevas tecnologías e Interdisciplinariedad en la comunicación del Patrimonio Cultural (pp. 92-103). AcerVOS; Universidad Pablo de Olavide; Universidad de Córdoba; Universidad Peruana Simón Bolívar. https://www.academia.edu/38037580/ Situaci $\% \mathrm{C}_{3} \% \mathrm{~B} 3 \mathrm{n}$ de las redes sociales en los museos del Per\%C3\%BA Claves y desaf\%C3\%ADos en Lima Arequipa y Cusco

Conseil International des Musées. (2019). Manuel des réseaux sociaux pour les comités de l'ICOM. https://icom.museum/wp-content/uploads/2019/10/ Social-media-guidelinesFR-1.pdf

De Garay, R. (2008). Art \& marketing: strategies de promotion et de diffusion de l'art. Ars Vivens.

Gran Teatro Nacional. (2020). GTN EN VIVO: Perfiles de públicos y visionado de experiencias escénicas. https://granteatronacional.pe/formacion/estudios-de-publicos

Gran Teatro Nacional. (2021, 30 de abril). Carmen. Ballet Nacional. Dir. artística Jimmy Gamonet [Imagen]. Facebook. https://shorturl.at/blyYo

Hootsuite. (2021). Digital 2021: Peru. https:// datareportal.com/reports/digital-2021-peru
Instituto de Opinión Pública. (2020, abril). Cambios en el consumo cultural en Lima-Callao 2009-2019. Boletín $N^{o}$ 169. http://repositorio.pucp. edu.pe/index/bitstream/handle/123456789/170283/IOP 111901 R4.pdf?sequence $=1$ \&isAllowed $=\mathrm{y}$

Instituto Nacional de Estadística e Informática. (2019). Patrimonio, bienes y servicios culturales 2016-2018. https:// www.inei.gob.pe/media/MenuRecursivo/publicaciones digitales/Est/ Lib1707/libro.pdf

Leal Jiménez, A. y Quero Gervilla, M. J. (2011). Manual de marketing y comunicación cultural (producto n. ${ }^{\circ} 44$ ). Observatorio Cultural del Proyecto Atalaya. https://www.bizkaia.eus/ home2/archivos/DPTO4/Temas/ producto 44 manual-de-marketing-y-comunicacion-cultural web. pdf?hash $=0023207$ f83f9356ae928f 4 fo13580512

MALI - Museo de Arte de Lima. (2020, 14 de abril). [\#QuédateEnCasa] ¿A quién no le ha pasado? [Imagen adjunta] [Actualización de estado]. Facebook. https://shorturl.at/amxX4

MALI - Museo de Arte de Lima. (2021, marzo). \#QuédateEnCasa. Recuerda que puedes seguir visitándolos en nuestras tres plataformas virtuales [Imagen adjunta] [Actualización de estado]. Facebook. https://shorturl.at/ijwV8

Ministerio de Cultura y Organización de las Naciones Unidas para la Educación, la Ciencia y la Cultura. (2015). 22 indicadores de la cultura para el desarrollo en Perú. Resumen analítico de Perú. http://www.infoartes. pe/wp-content/uploads/2014/12/ libro-22-Indicadores-de-Cultura-para-el-Desarrollo2.pdf 
Ministerio de Cultura y Municipalidad de Lima. (2020). Informe sobre el impacto del estado de emergencia por el COVID-19 en el sector de las artes, museos e industrias culturales y creativas. Resultados generales. https://cdn.www.gob.pe/uploads/ document/file/790476/Informe ok 05JUN20.pdf

Ministerio de Cultura. (2021). ¿Cómo vamos en el consumo de Teatro en el Perú? Análisis de indicadores de asistencia a espectáculos de teatro entre el 2016 y el 2019. https://cdn.www.gob. pe/uploads/document/file/1817472/ Cómo\%20vamos\%20en\%20el\%20 consumo $\% 2$ ode $\% 20$ teatro $\% 20$ en\%2oel\%2oPerú.pdf.pdf

Museo de Arte de Lima. (2018). Hacia un museo sostenible. Oferta y demanda de los museos y centros expositivos de Lima. https://mali.pe/portfolio-item/ proyecto-de-investigacion-para-mejorar-la-sostenibilidad-de-los-mu$\underline{\text { seos/ }}$ 